\title{
A matter of lubrication
}

A 64-year-old male was admitted to a pulmonary unit with chronic cough, right moderate thoracic pain and abnormal chest radiography. He had a 40-pack-year smoking history ending 1 year previously, when a laryngectomy and a hemithyroidectomy were performed for cancer, followed by neck radiotherapy. He had a history of COPD and arterial hypertension.

\section{Case referral}

Physical examination was unremarkable except for the presence of crackles at left lung base. Laboratory studies and blood chemistries were within normal limits. Erythrocyte sedimentation rate was $18 \mathrm{~mm}$ per hour. The patient underwent chest radiography and computed tomography (CT; figures 1 and 2 ).

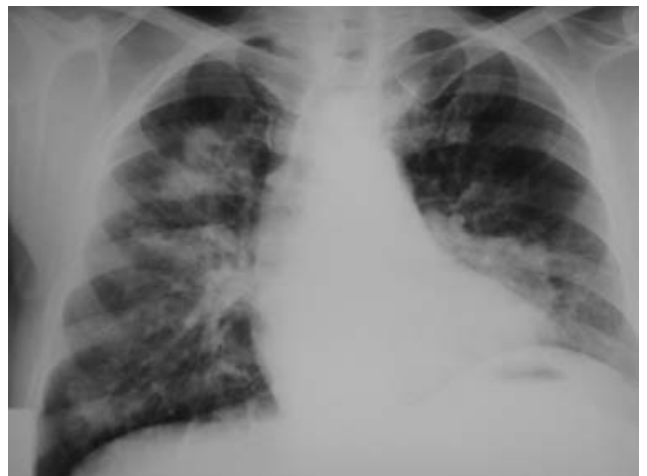

F. Norrito ${ }^{1}$

V. Cusimano ${ }^{2}$

G. Lipani ${ }^{3}$

G. Madonia ${ }^{1}$

\section{Figure 1}

Chest radiography.

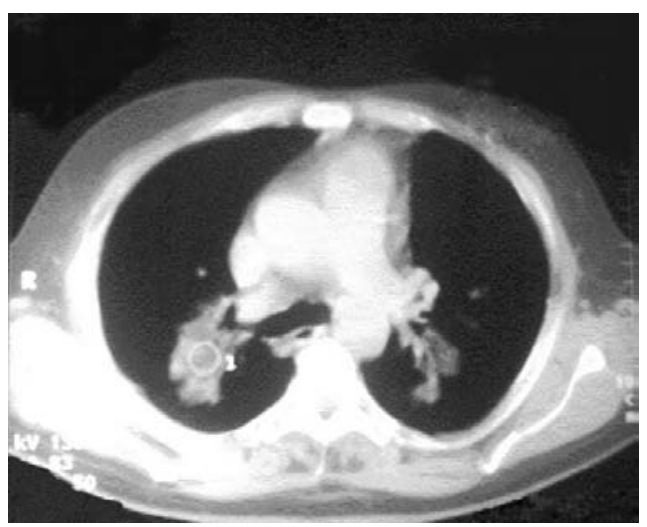

Correspondence:

G. Madonia

Unit of Respiratory

Physiopathology

Ospedale Civico ARNAS

Palermo

90143 Italy

Fax: 390916663572

E-mail:mad53@inwind.it
Figure 2

CT scan of the chest.

\section{Task 1}

Interpret the radiograph and CT scan. 


\section{Answer 1}

Bilateral alveolar consolidation can be seen predominantly in the posterior zones of the lower lobes and in perihilar dislocation, with variable irregular margination and low density indexes $(-30 /-70$ Hounsfield Units (HU)).

Next, flexible bronchoscopy was performed but no airway abnormalities were seen. Cytological and bacteriological analysis of aspirated secretions from bronchial washing showed only macrophages and leukocytes, with no tumoral cells or significant microbial forms.

Right video-assisted thoracic surgery (VATS) with lung biopsy was then performed. The histopathological findings are shown in figure 3.

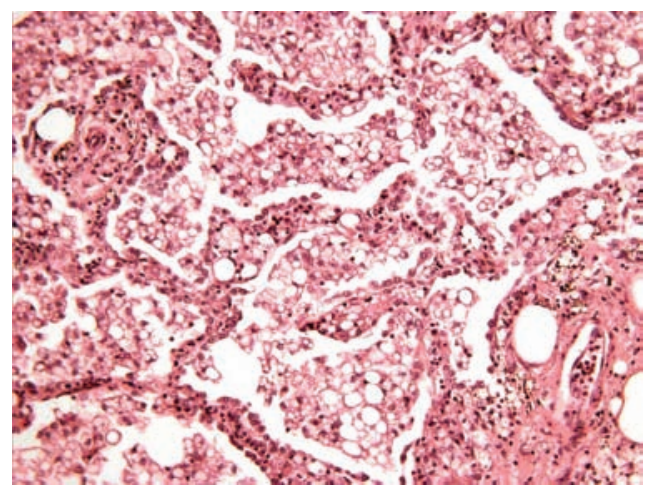

\section{Figure 3}

Lung biopsy specimen.

Task 2

How would you interpret the histological results?

\section{Answer 2}

The histological results show alveolar and interstitial spaces infiltrated by macrophages containing lipoid vacuoles. Inflammatory infiltration of interstitial structures by lymphocitic and plasma cells can be seen (haematoxylin eosin, 20).

Subsequently, a detailed history of the patient's drug use revealed that, since the laryngectomy, the patient had taken to instilling in his tracheostomy a rhinological oily solution containing Vaseline, twice daily. He explained that he enjoyed the refreshing sensation in his airways.

\section{Task 3 \\ How many types of lipoid pneumonia are there?}

\section{Task 4 \\ Based on the background information presented here, how would you treat this case?}




\section{Answer 3}

Lipoid pneumonia is a rare pulmonary disease that has no typical radiological appearance, and can thus imitate many other lung diseases. Lipoid pneumonia is usually classified into two major groups, depending on whether the source of oil/fat in the respiratory tract is exogenous or endogenous [1-3].

\section{Answer 4}

The treatment of lipoid pneumonia is generally conservative and essentially consists of preventing further exposure. Once exposure has stopped, the radiographic findings improve or remain stable in the majority of patients. Systemic steroid treatment and therapeutic bronchoalveolar lavage have been advocated in some cases, but no benefit has been definitively shown $[1,2,4]$.

\section{Discussion}

Lipoid pneumonia has been a recognised diagnosis since 1925 [5]. It is a rare condition that can mimic many other diseases, as its clinical and radiographical characteristics are nonspecific. This makes it a very difficult diagnosis to make without a great deal of suspicion. Lipoid pneumonia is usually classified into two major groups depending on the source of fatty substances as follows.

Exogenous lipoid pneumonia (ELP) is caused by inhalation, aspiration, respiration and even intravenous injection of oily substances of animal, vegetable or mineral nature. Owing to its high viscosity, this oily/fatty material supresses the cough reflex and, once in the alveoli, provokes a foreign body-type inflammatory reaction $[1,2,6]$.

Endogenous (or cholesterol) lipoid pneumonia is a condition that can result from the leakage of lipids from destroyed alveolar cell walls (as in suppurative processes or airway obstructive lesions), from lipid storage disorders (as in Niemann-Pick disease) or from idiopathic processes [7].

Clinically, ELP has traditionally been considered a chronic disease caused by repeated aspiration or inhalation of small quantities of fatty substances. However, massive aspiration of oily material can result in an acute form of pneumonia with severe or fatal intoxication, as can happen to fire-eaters, or, in some circumstances, multiple organ failure after an intravenous injection of lamp oil [8]. Most patients are elderly, in the sixth or seventh decade of life $[1,2]$.
Almost half of patients with ELP are without symptoms on presentation, and are only identified because of an abnormality seen on chest radiography $[6,9-11]$. Patients with symptoms commonly present with chronic cough or dyspnoea. In addition, fever, weight loss, chest pain and haemoptysis are seen less often. The physical examination may be normal, or may reveal crackles or wheezes.

In a retrospective multicentre study in France, the commonest aetiological circumstances involved administration of liquid paraffin for the treatment of refractory constipation and the nasal instillation of oily products for chronic rhinopharyngeal diseases. The oil responsible was almost always a mineral oil (paraffin or Vaseline) [3].

Predisposing factors for aspiration, such as advanced age, debilitated state, gastrooesophageal reflux and neurological disorders that interfere with cough or swallowing have all been associated with cases of ELP. However, many cases occur in patients without any predisposing conditions $[1-3,6]$.

The CT scan is helpful in the diagnosis of ELP, as it can support the diagnosis by detecting the presence of lipid deposits in lung masses. Although this finding is not present in all cases, a negative density (-150- -30 HU) is highly suggestive of intrapulmonary fat and a diagnosis of lipoid pneumonia [6]. Obviously, these attenuation values are higher than that of pure fat, because of the cellular inflammatory infiltration. In addition, the examination of sputum and bronchoalveolar lavage can show separate fat globules and macrophages filled with fat droplets. However, if the diagnosis remains uncertain, the final diagnosis is based on a histological examination of a lung biopsy.

In the current patient, the diagnosis of exogenous lipoid pneumonia could have been made on the basis of characteristic findings on CT scan and evidence of exposure to mineral oil. However, initial investigations (on the use of rhinological drops) were incomplete and a biopsy sample was presumed necessary to exclude a neoplastic disease. Unfortunately, the sample could be examined only with haematoxylin-eosin, because no cryostat sections were available to perform other fat staining (Oil Red or Sudan Black). Moreover, bronchoalveolar lavage was not carried out because the fundamental clinical suspicion was that of a lung cancer, since the patient had been a heavy smoker. The incomplete and detail-lacking history was misleading, reinforcing the old adage: "good history, good diagnosis". 


\section{References}

1. Wright B, Jeffrey P. Lipoid pneumonia. Semin Respir Infect 1990; 5: 314-321.

2. Spickard A, Hirschmann J. Exogenous lipoid pneumonia. Arch Intern Med 1994; 154: 686-692.

3. Gondouin A, Manzoni P, Ranfaing E, et al. Exogenous lipoid pneumonia: a retrospective multicentre study of 44 cases in France. Eur Respir J 1996; 9: 1463-1469.

4. Chin NK, Hui KP, Sinniah R, Chan TB. Idiopathic lipoid pneumonia in an adult treated with prednisolone. Chest 1994; 105: 956-957.

5. Laughlen GF. Studies on pneumonia following nasopharyngeal injections of oil. Am J Pathol 1925; 1: 407-415.

6. Adkins D, Bensadoun SE. An 85-year-old man with a lung mass. Chest 2004; 125: 1121-1123.

7. Nicholson AG, Wells AU, Hooper J, Hansell DM, Kelleher A, Morgan C. Successful treatment of endogenous lipoid pneumonia due to Niemann-Pick type B disease with whole-lung lavage. Am J Respir Crit Care Med 2002; 165: 128-131.

8. Houben MHMG, Drent M, Jacobs JA, Schrey G, Ramsay G. Multiple organ dysfunction syndrome (MODS) after an intravenous injection of lamp oil (liquid paraffin). Intensive Care Med 1998; 1: 87-88.

9. Brechot JM, Buy JN, Laaban JP, Rochemaure J. Computed tomography and magnetic resonance findings in lipoid pneumonia. Thorax 1991; 46: 738-739.

10. Lee KS, Muller NL Hale V, Newell JD Jr, Lynch DA, Im JG. Lipoid pneumonia: CT findings. J Comput Assist Tomogr 1995; 19: 48-51.

11. Lee JS, Im JG, Song KS, Seo JB, Lim TH. Exogenous lipoid pneumonia: high-resolution CT findings. Eur Radiol 1999; 9 : 287-291. 